\title{
Folding Properties of Two-Dimensional Deployable Membrane Using FEM Analyses
}

\author{
By Yasutaka SATOU ${ }^{1)}$ and Hiroshi FurUYA ${ }^{1)}$ \\ ${ }^{1)}$ Department of Built Environment, Tokyo Institute of Technology, Yokohama, Japan
}

(Received July 17th, 2009)

Folding FEM analyses are presented to examine folding properties of a two-dimensional deployable membrane for a precise deployment simulation. A fold model of the membrane is proposed by dividing the wrapping fold process into two regions which are the folded state and the transient process. The cross-section of the folded state is assumed to be a repeating structure, and analytical procedures of the repeating structure are constructed. To investigate the mechanical properties of the crease in detail, the bending stiffness is considered in the FEM analyses. As the results of the FEM analyses, the configuration of the membrane and the contact force by the adjacent membrane are obtained quantitatively for an arbitrary layer pitch. Possible occurrence of the plastic deformation is estimated using the Mises stress in the crease. The FEM results are compared with one-dimensional approximation analyses to evaluate these results.

Key Words: Solar Sail, Fold, FEM, Membrane, Crease

\section{Nomenclature}

$\begin{array}{lll}h & : \text { layer pitch } \\ s & : \text { body fixed system } \\ x, y, z & : \text { global coordinate system } \\ t & : \text { membrane thickness } \\ E_{i}^{j} & : \text { definition of edge } \\ f_{l} & : \text { edge load } \\ P & : \text { contact force by adjacent membrane } \\ \kappa & : \text { curvature of crease } \\ \theta & : \text { deflection angle } \\ \theta_{0} & : \text { deflection angle at } s=a+b \\ E & : \text { Young's modulus } \\ I & : \text { moment of inertia of area } \\ T_{a+b} & : \text { in-plane force at } s=a+b \\ Q_{a+b} & : \text { shear force at } s=a+b \\ b & : \text { membrane width } \\ u_{0}, w_{0} & : \text { displacement of neutral line } \\ \sigma_{\text {Mises }} & : \text { Mises stress } \\ n & : \text { calculation step }\end{array}$

\section{Introduction}

There is currently much interest in the use of two-dimensional deployable membranes for several lightweight space structures: solar sails, large aperture antennas, sunshields, and solar power satellites. Since these deployable structures are consists of thin and large membranes, the membrane requested to be folded on the ground and deployed in the space. Hence, the retraction and the deployment of the membrane are the key technical issues to realize the two-dimensional deployable membrane.

The deployment dynamics of the membrane is simulated in several researches. It was indicated with the numerical analyses by Miyazaki ${ }^{1}$ that the fold pattern determines the dynamical properties of the deployment. The proposed numerical calculation code considers a wrinkle/slack model. However, the folding properties, which are the configuration of the crease, the elastic restoring force of fold lines, the plastic deformation of creases, wrinkles in the folded state, and the contact force by the adjacent membrane, are not exactly evaluated; these parameters are requested for the precise deployment simulation.

Retraction mechanisms have been developed by the authors $^{2)}$ to manufacture and retract a large membrane automatically. The main concept of the retraction mechanisms is to wrap and fold the membrane using tensile forces and a polygonal center hub. As the extended retraction mechanisms, guided-pin mechanisms have been also proposed by the authors ${ }^{3)}$. Retraction experiments using the guided-pin mechanisms indicated that the packaging density and the precision of the retraction are determined by the folding properties: the configuration of the crease and the contact force.

Folding analyses were performed in several researches. Woo performed creasing simulations to predict the creased geometry using two-dimensional plane strain elements ${ }^{4}$. Kawaguchi demonstrated large deformation analyses for a membrane element ${ }^{5}$. However, these research works didn't treat the mechanical condition of the wrapping fold membrane, which folded several times, although the mechanical condition determines the folding properties. The bending stiffness was not considered; it is dominant in the folded state. The effects of the contact force were not treated although the effects determine the folding properties.

In this paper, folding FEM analyses are presented to examine the folding properties considering the mechanical condition of the folded state, and the bending stiffness, and the effects of the contact force. A fold model is proposed by investigating the folding process of the membrane retracted by 
the guided-pin mechanisms. Analytical procedures of the folded state are constructed for FEM. The numerical results are discussed quantitatively about the configuration of the crease, the Mises stress, the plastic deformation, and the contact force by the adjacent membrane. The results of the FEM analyses are compared with one-dimensional approximation analyses to evaluate these results.

\section{Overview of Guided-Pin Mechanisms}

To retract a membrane automatically on the ground, a folding apparatus is requested. To this end, retraction mechanisms are proposed by the authors. The main concept of the retraction mechanisms is to wrap and fold the membrane with the tensile forces and the polygonal center hub. Mixed spiral fold (lower hand of Fig.1), which consists of Z-folds and spiral folds, was obtained in the retraction experiments with few wrinkles and high packaging density.

To fold an arbitrary-sized membrane, guided-pin (upper hand of Fig.1) was proposed. It was indicated that the guided-pin generates the fold line with the out-of-plane enforced displacement and the tensile forces by the friction between the membrane and the guided-pin.

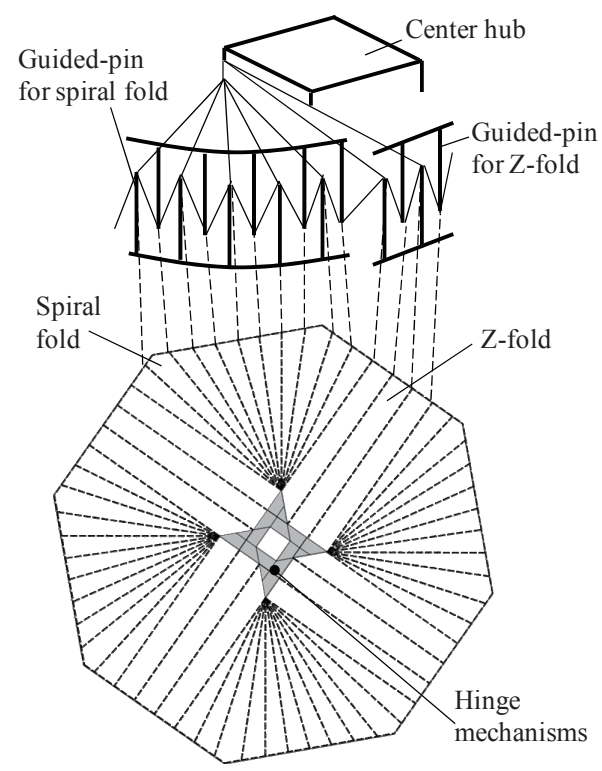

Fig. 1. Overview of guided-pin retraction mechanisms.

\section{Fold Model for Numerical Simulation}

\subsection{Fold model of membrane}

A fold model of the membrane retracted by the guided-pin mechanisms is proposed considering the mechanical properties of the wrapping fold process. The fold model is shown in Fig.2. As shown in the figure, the membrane is folded by the guided-pin, and is wrapped around a square center hub. In the figure, a part of Z-fold area is illustrated for the simplified illustration. As shown in the figure, the fold condition is divided into two regions, which are region 1 and region 2. The aim of the division is to treat the mechanical condition of the wrapping fold membrane simply. In the region 1 , the membrane is fully folded and wrapped around the center hub, and the region is the folded state. In the region 2 , the membrane is being folded by the guided-pin, and is being wrapped around the center hub, and the region is the transient process. In this paper, we focus on the folded state of the membrane to examine the folding properties.

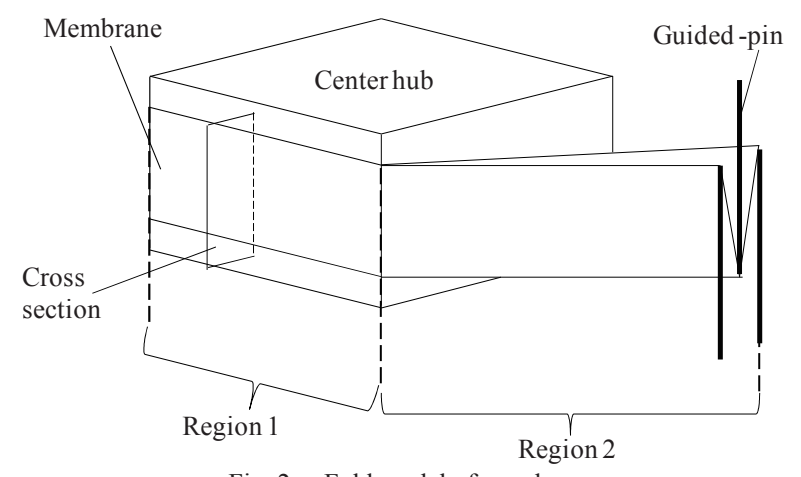

Fig. 2. Fold model of membrane.

The cross-section of the membrane, which is fully folded and wrapped around the center hub, is assumed to be a repeating structure as shown in Fig.3. In the figure, $h$ indicates the layer pitch of the folded state. On the assumption of the repeating structure, the selected area is investigated in numerical analyses. In fact, the membrane is retracted by the wrapping tensile forces and the contact force by adjacent membrane. When the assumption of the repeating structure is introduced, the wrapping tensile force is impossible to apply to the membrane. Thus, the corresponding contact force is applied to the membrane.

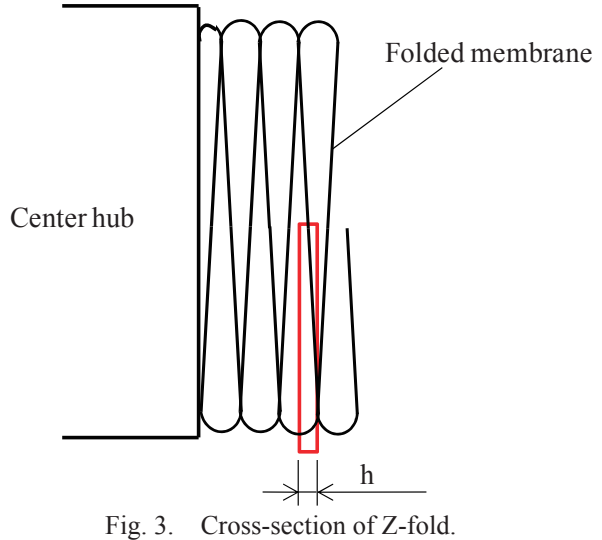

\subsection{Initial configuration of fold model}

Figure 4 indicates the initial configuration of the fold model. As shown in the figure, a strip membrane is introduced to evaluate FEM results by comparing with the results of one-dimensional approximation analyses, and as the first stage of the folding FEM analyses. Since the initial configuration of the practical membrane is flat, the initial configuration of the fold model is also flat. In the figure, $t$ is the membrane thickness, and $s$ is the body fixed system. 


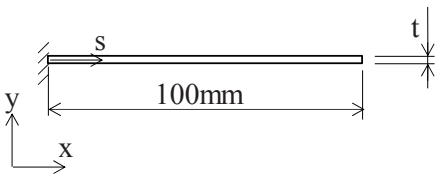

(a) Side view of $x-y$ surf.

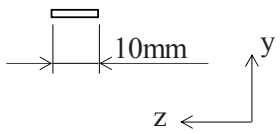

(b) Side view of $y-z$ surf.

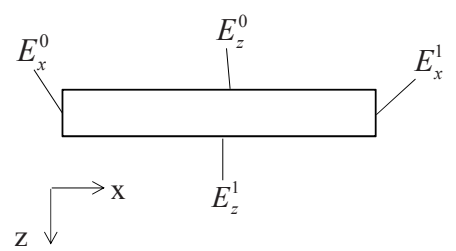

(c) Plane view

Fig. 4. Initial configuration of fold model.

\subsection{Analytical procedures}

Analytical procedures of the repeating model are constructed for numerical simulations. The analytical procedures are shown in Fig.5. A broken line indicates the initial configuration of the membrane. In step 1, an edge load $f_{1}$ is applied to $E_{x}{ }^{l}$ to bend the membrane. The edge load corresponds to the folding load of the guided-pin. On the assumption of the repeating structure, $E_{x}{ }^{0}$ is fully built in, and a moment of $E_{x}{ }^{1}$ is 0 . In step 2, a contact force is applied to the membrane by a plate. Since the contact area between the membrane and the plate is unknown, boundary condition of the displacement of the plate is applied, and the contact force is obtained. On the assumption of the repeating structure, $E_{x}{ }^{l}$ is $h / 2$ away from $y-z$ surface.

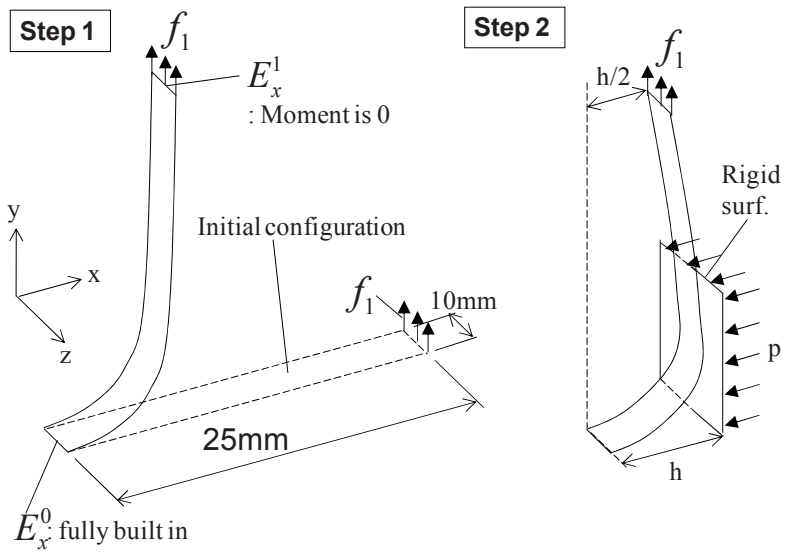

(a) Apply edge load $f_{1}$

(b) Apply contact force

Fig. 5. Analytical procedures of repeating model.

\section{One-Dimensional Approximation Analyses}

To approximate the proposed repeating model, one-dimensional fold model was introduced using Elastica by the authors ${ }^{6}$. Figure. 6 indicates the proposed one-dimensional fold model.

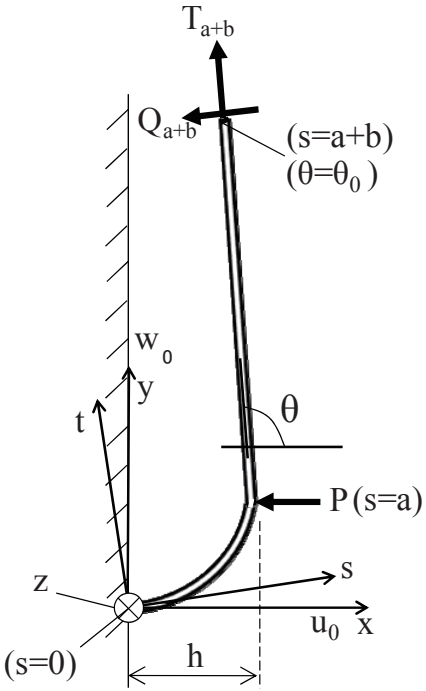

Fig. 6. One-dimensional fold model.

The governing equation of the one-dimensional model is described as the distribution of the curvature from $\theta=0$ to $\pi / 2$ as Eq. (1).

$\kappa=\frac{d \theta}{d s}=\sqrt{\frac{2}{E I}} \sqrt{P\left(\cos \theta-\cos \theta_{0}\right)+T_{a+b}\left\{1-\cos \left(\theta-\theta_{0}\right)\right\}-Q_{a+b} \sin \left(\theta-\theta_{0}\right)}$

Using the Eq. (1), the layer pitch $h$ (Fig.3, 6) and the Mises stress of the crease $(\mathrm{s}=0)$ are derived as,

$h=\sqrt{\frac{E I}{2}} \int_{0}^{\pi / 2} \frac{\cos \theta}{\sqrt{P\left(\cos \theta-\cos \theta_{0}\right)+T_{a+b}\left\{1-\cos \left(\theta-\theta_{0}\right)\right\}-Q_{a+b} \sin \left(\theta-\theta_{0}\right)}} d \theta$

$$
\sigma_{\text {Mises }}=\sqrt{\frac{6 E}{t b}} \sqrt{\left(P+T_{a+b}\right)\left(1-\cos \theta_{0}\right)+Q_{a+b} \sin \theta_{0}}
$$

Eq. (2) shows the layer pitch for arbitrary contact force $P$ (Fig.5b, 6). Eq. (3) estimates possible occurrence of the plastic deformation of the membrane by comparing with the yield stress of the membrane.

\section{Details of FEM Analyses}

FEM analyses are performed for the repeating model with ABAQUS ${ }^{7)}$, commercial software. To investigate the mechanical properties of the crease in detail, the bending stiffness is considered by using 4-node thin shell elements (S4R5). As the deformation of the membrane is geometrical nonlinear, NLgeom option is applied. Contact analyses are performed between the membrane and the plate. The finite element mesh is indicated in Fig.7. For the membrane, Young's modulus is $3.19 \mathrm{GPa}$, and the Poison's ratio is 0.300 .

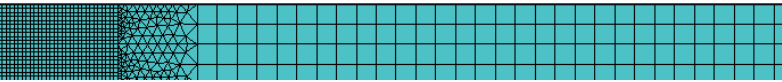

Fig. 7. Finite element mesh for fold model. 


\section{Analytical Results and Discussions}

\subsection{Deformation process of fold model}

To improve precision of a deployment dynamics, configurations of the folded membrane is requested. Figure 8 shows the deformation process of the fold model. The membrane thickness is $10 \mu \mathrm{m}$, the layer pitch is $2 \mathrm{~mm}$, and the edge load is $0.00001 \mathrm{~N} / \mathrm{mm}$. $n$ indicates the calculation step. The calculation step of the initial configuration is 0 . The edge load is applied to the $E_{x}{ }^{l}$ of the initial configuration, and the membrane deforms as shown in the figure. When the calculation step $n=301$, the membrane is bended with the edge load. Then, the contact force is applied to the membrane indicated as $n=301$. Finally, the folded configuration ( $n=$ 392 ) is obtained. As shown in the figure, the membrane is creased by the contact force mainly.

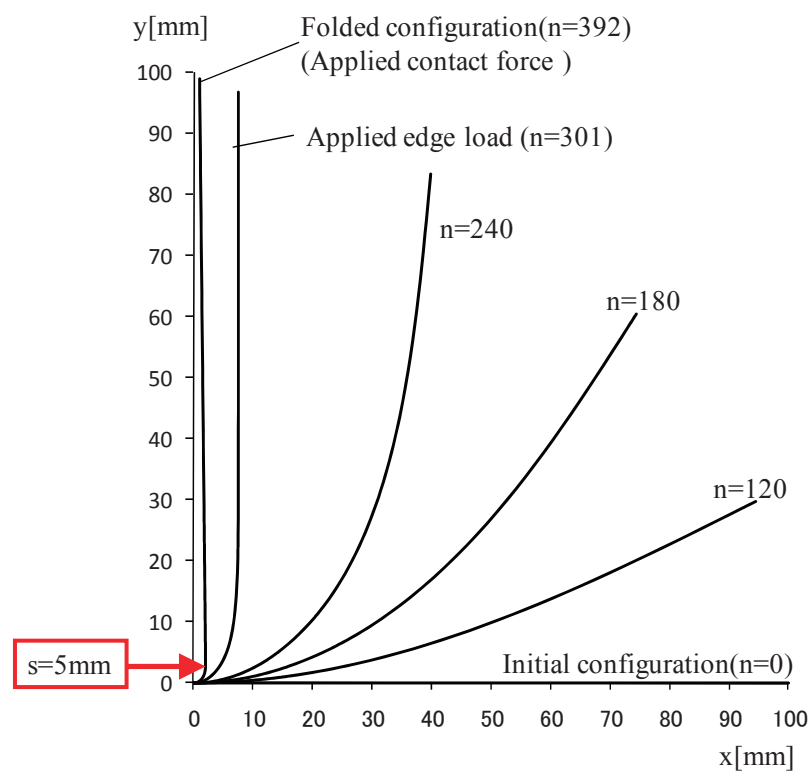

Fig. 8. Configuration of fold model $(t=10 \mu \mathrm{m}, h=2 \mathrm{~mm}, f=$ $0.00001 \mathrm{~N} / \mathrm{mm})$

The corresponding curvature is indicated in Fig.9. As shown in the figure, the curvature is localized around $s=0$. Hence, the crease region is recognized to be the region which is from $s=0 \mathrm{~mm}$ to $s=5 \mathrm{~mm}$. The corresponding region is indicated in the Fig.8. When the solar cells are attached to the membrane, damages to the cells will be reduced by avoiding the crease region.

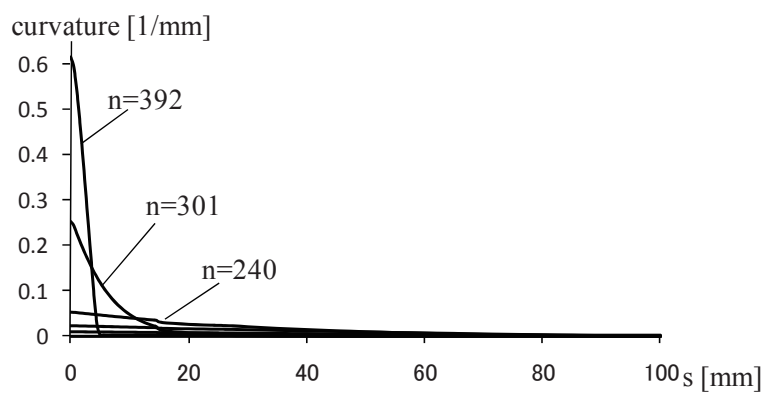

Fig. 9. Curvature of fold configuration $(t=10 \mu \mathrm{m}, h=2 \mathrm{~mm}, f=$ $0.00001 \mathrm{~N} / \mathrm{mm})$.

\subsection{Crease configuration for contact force}

The results of the FEM analyses are compared with the results of the one-dimensional approximation analyses. Figure 10 and 11 indicate the configuration of the crease and the distribution of the curvature, each other. Broken lines indicate the results of the one-dimensional analyses, and continuous lines indicate the results of the FEM analyses. The parameters used in the one-dimensional analyses are indicated in Tab.1. The results of the one-dimensional analyses are described from $\theta=0$ to $\pi / 2$, because the Eq. (1) is applicable for the range of the deflection angle. The applied contact forces to the one-dimensional model are determined by the results of the FEM analyses, which are folded to $2 \mathrm{~mm}, 3 \mathrm{~mm}$, and $4 \mathrm{~mm}$. As shown in the figure, the smaller layer pitch, the larger curvature is obtained. The differences between the results of the one-dimensional analyses and that of the FEM analyses are caused by the contact force; the contact force of the one-dimensional analyses is the concentrated force, and that of the FEM analyses is the distributed force. The difference of each maximum curvature is about $5 \%$.

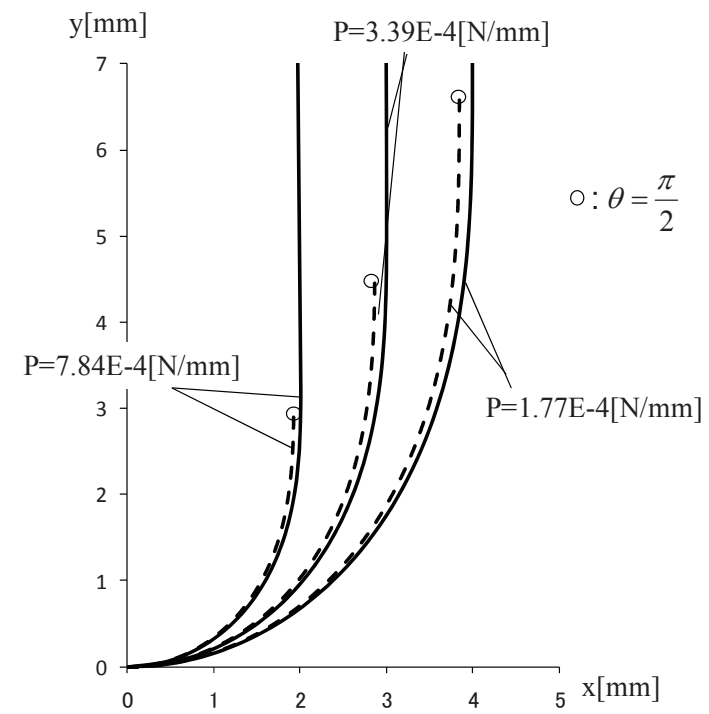

Fig. 10. Configuration of crease $\left(t=25 \mu \mathrm{m}, f=5.00 \times 10^{-5} \mathrm{~N} / \mathrm{mm}\right)$.

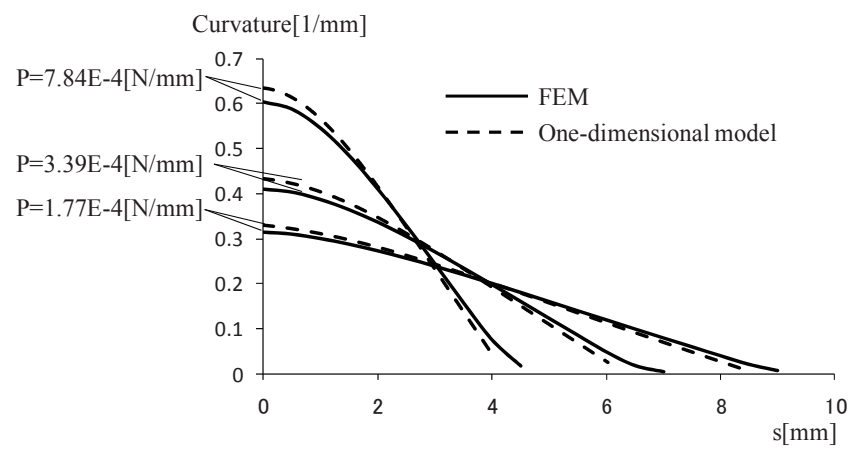

Fig. 11. Curvature around fold line $(t=25 \mu \mathrm{m}, f=0.00005 \mathrm{~N} / \mathrm{mm})$. 
Table 1. Parameters of numerical analyses.

\begin{tabular}{|l|l|}
\hline shear force in $s=a+b, Q_{a+b}$ & $0.00 \mathrm{~N} / \mathrm{mm}$ \\
\hline deflection angle in $s=a+b, \theta_{0}$ & $\pi / 2$ \\
\hline membrane width, $b$ & $1.00 \mathrm{~mm}$ \\
\hline membrane length, $a+b$ & $100 \mathrm{~mm}$ \\
\hline membrane thickness, $t$ & $25.0 \mu \mathrm{m}$ \\
\hline in-plane force in $s=a+b, T_{a+b}$ & $2.00 \times 10^{-4} \mathrm{~N} / \mathrm{mm}$ \\
\hline Young's modulus, $\mathrm{E}$ & $3.19 \mathrm{GPa}$ \\
\hline
\end{tabular}

\subsection{Effects of layer pitch on fold configuration}

To examine the relationships between the layer pitch and the region of the crease in detail, the region of the crease is estimated as shown in Fig.12. As shown in the figure, the region of the crease is directly proportional to the layer pitch. The maximum curvature $(s=0)$ is also plotted in Fig.13. The figure shows that the maximum curvature is inversely proportional to the layer pitch.

region of crease in $s[\mathrm{~mm}]$

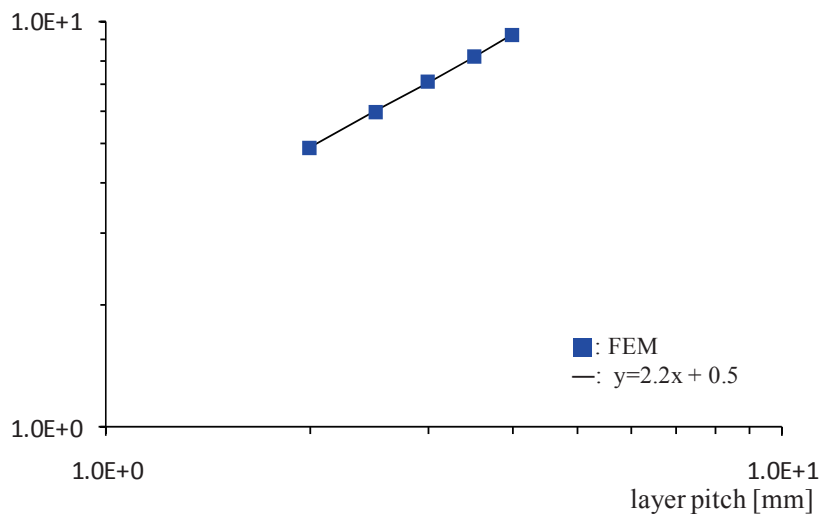

Fig. 12. Region of crease vs layer pitch.

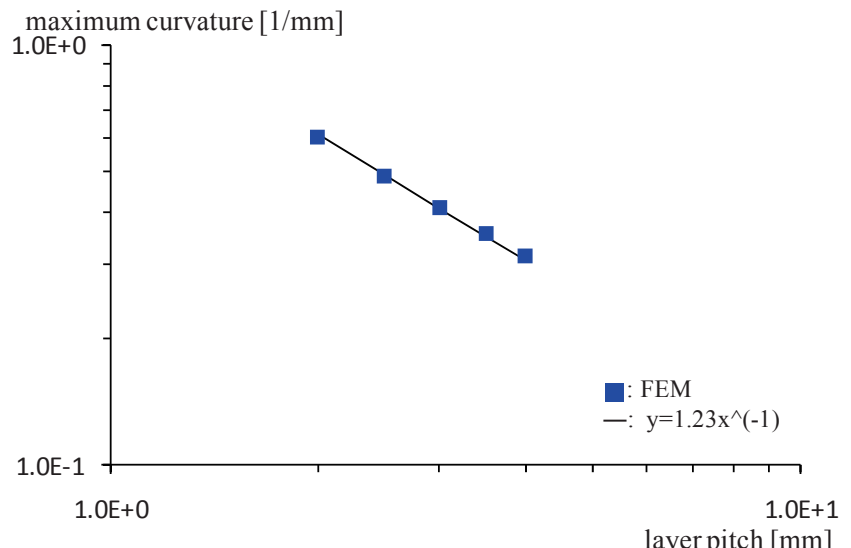

Fig. 13. Maximum curvature vs layer pitch

\subsection{Maximum Mises stress of membrane}

To estimate the yield point of the membrane, and to predict the stress in the crease, maximum Mises stress in the crease at $s=0$ is examined. Figure 14 indicates maximum Mises stress of the membrane for the layer pitch. The dots are the results of FEM, and the continuous lines are the results of the one-dimensional analyses. As shown in the figure, the values of the results of the one-dimensional analyses are larger than that of the FEM. As the reason of the results, the existence of Poison's ratio is supposed. Because FEM is two-dimensional analysis, the bending stiffness of the one-dimensional fold model of Eq. (3) is larger than that of the FEM. To estimate the possible occurrence of the plastic deformation, the Mises stress is compared with the yield stress of the membrane. For example, when the yield stress is $69 \mathrm{MPa}$, the membrane of upper two dots $(h=2 \mathrm{~mm}$, and $2.5 \mathrm{~mm}$ of $t=100 \mu \mathrm{m})$ will occur the plastic deformation. Hence, the possible occurrence of the plastic deformation is predictable using analytical results.

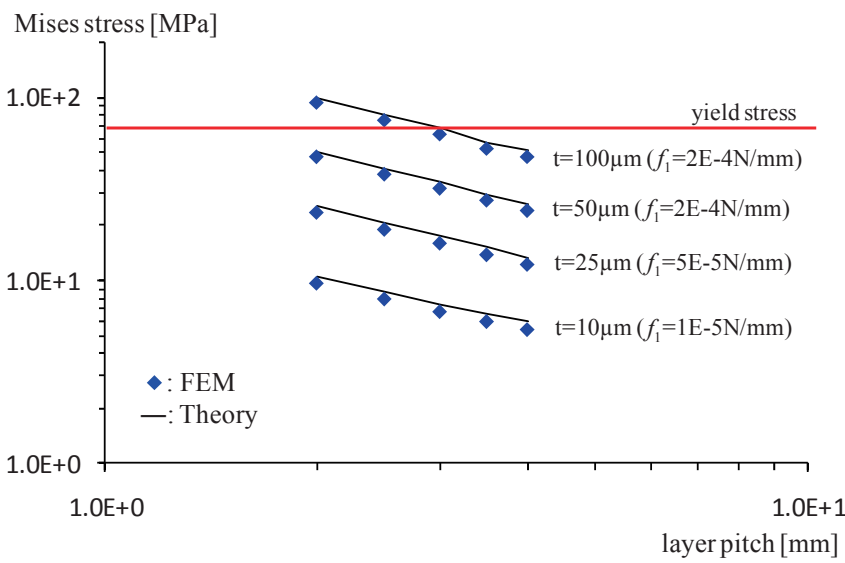

Fig. 14. Maximum Mises stress vs layer pitch.

Figure 15 shows the maximum Mises stress of membrane for the membrane thickness. The dots are the results of FEM, and the continuous lines are the results of the one-dimensional fold analyses. As shown in the figure, when the membrane is folded to an arbitrary layer pitch, the thick membrane has more Mises stress.

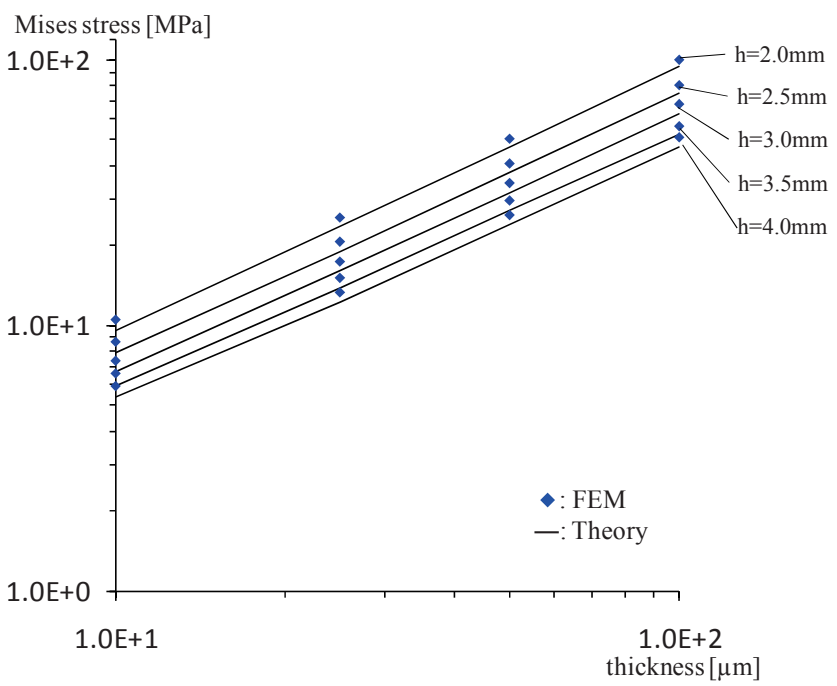

Fig. 15. Maximum Mises stress vs membrane thickness. 


\subsection{Contact forces}

Contact forces by adjacent membrane are investigated to examine the contact force for an arbitrary layer pitch. Figure 16 indicates the results of the analyses. The dots are the results of FEM, and the continuous lines are the results of the one-dimensional analyses. The contact forces are obtained quantitatively by applying the FEM analyses. As shown in the figure, the thick membrane and narrow layer pitch has more contact force. The results are in good agreement between the FEM and the one-dimensional analyses. Looking carefully, the larger layer pitch, and the thicker membrane thickness, the larger difference between these results exists. As the reason of the results, it is supposed that the effects of the edge load is not negligible opposed to the contact force.

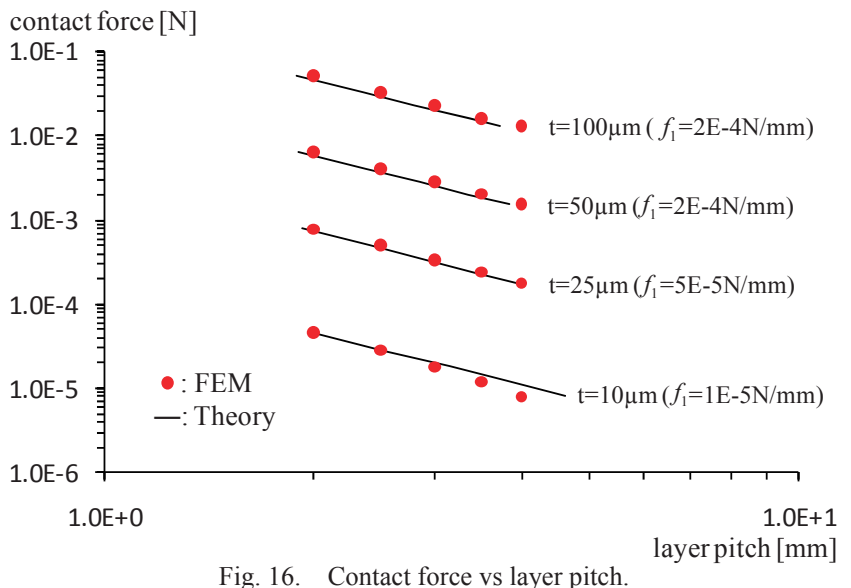

\section{Conclusions}

Folding properties of a two-dimensional deployable membrane were examined by FEM analyses. A fold model of the membrane retracted by guided-pin mechanisms was proposed by dividing the wrapping fold process into two regions which are the folded state and the transient process. The cross-section of the folded state was assumed to be a repeating structure, and the contact force was applied to fold the membrane. The analytical procedures of the repeating model were constructed for FEM analyses. As the results of the FEM analyses, the configuration of the crease and the contact force were obtained quantitatively, and the effects of the layer pitch were investigated. The FEM results were compared with the results of one-dimensional analyses to evaluate these results. Using the Mises stress in the crease, the possible occurrence of the plastic deformation was estimated.

\section{References}

1) Miyazaki, Y.: Wrinkle/Slack Model and Finite Element Dynamics of Membrane, International Journal for Numerical Methods in Engineering, 66(7)(2006), pp.1179-1209.

2) Furuya, H. and Satou, Y.: Deployment and Retraction Mechanisms for Spinning Solar Sail Membrane, Proceedings of 49th AIAA/ASME/ASCE/AHS/ASC Structures, Structural Dynamics and Materials Conference, Schaumburg, Illinois, (2008), pp.1-13.

3) Satou, Y. and Furuya, H.: Generating Fold Line with Guided-pin for Wrapping Large Membrane, Proceedings of the 50th JSASS/JSME Structures Conference, Kitakyuusyuu, (2008), pp.220-222(in Japanese).

4) Woo, K. and Jenkins, C. H.: Effective Modulus of Creased Thin Membrane, Proceedings of 47 th AIAA/ASME/ASCE/AHS/ASC Structures, Structural Dynamics and Materials Conference, Newport, Rhode Island, (2006), pp.1-13.

5) Ito, K. and Kawaguchi, K.: Folding Analysis of Membrane Structures Part 2, Summaries of technical papers of Annual Meeting Architectural Institute of Japan, (1996), pp.901-902(in Japanese).

6) Satou, Y. and Furuya, H.: Retractability of Two-dimensional Deployable Membrane for Spinning Solar Sail, Proceedings of 26th International Symposium on Space Technology and Science, Hamamatsu, (2008), pp.1-6.

7) ABAQUS user's manual, Version 6.8, ABAQUS Inc., 2008. 